\title{
A Case of Thoracic Empiyemia Secondary to Serratia rubidaea
}

\author{
Emel Uzunoglu ${ }^{1}$, Ahmet Melih Sahin ${ }^{2}$, Ali Celik ${ }^{3}$, Cuneyt Cal ${ }^{1}$, Alptekin Tosun $^{4}$ \\ ${ }^{1}$ Giresun University, Medical Faculty, Medical Microbiology Department, Giresun \\ ${ }^{2}$ Giresun University, Prof. Dr. Ilhan Ozdemir Education and Research Hospital, Medical Microbiology and Infectious Disease \\ Clinic, Giresun \\ ${ }^{3}$ Giresun University, Prof. Dr. Ilhan Ozdemir Education and Research Hospital, Thoracic Surgery Clinic, Giresun \\ ${ }^{4}$ Giresun University, Medical Faculty, Radiology Department, Giresun
}

Received: 4 August 2017 Accepted: 15 August 2017, Published online: 28 August 2017

(C) Ordu University Institute of Health Sciences, Turkey, 2017

\begin{abstract}
Serratia rubidaea is an oportunistic pathogen which is a member of unusual Serratia species. This is the the first thoracic empyema case report caused by S. rubidaea which is isolated from the pleural fluid of an immunocompetent patient. There is no concensus about the natural habitat of $S$. rubidaea and the infection was community-based in our case. Here we discussed both the identification and the antibiyogram susceptibility of S. rubideae which needs a special attention hence some common commercial identification systems are lack of carbon utilisation tests. Overlooked laboratory diagnosis and ampric antibiotic treatment may cause life treating infections due to naturally occurring Amp C $\beta$-lactamases of unusual Serratia rubideae. Therefore, further studies are needed to identify both the clinical presentation and source of the agent.

Key words: Serratia rubideae, pleural effusion, thoracic ampitema, Serratia spp.
\end{abstract}

Address for correspondence/reprints:

Emel Uzunoglu

Telephone number: +90 (505) 7785529

E-mail: emeluzunoglu@yahoo.com

DOI: $10.19127 / \mathrm{mbsjohs.332727}$

\section{Introduction}

Genus Serratia includes eighteen species. The majority of Serratia infections are caused by Serratia marcescens and Serratia liquefaciens complex. Serratia rubidaea (S. rubidaea) is an oportunistic pathogen which is a rare cause of Serratia-related infections (Bonnin et al.,2015). Till now, it has generally been isolated from sputum or wound sites of debilitated hospitalized patients (Menezes et al., 2004; Gentille et al., 2014; Farmer et al., 1985). While other Serratia species are associated with insects, the natural habitat of $\mathrm{S}$. rubideae is not known yet (Litterio et al., 2012). Herein we report the first thoracic empyema case in the literature secondary to S. rubidaea isolated from the pleural fluid of an immunocompetent patient. 


\section{Case Report}

A 42 - year - old male patient who had been using amoxicillin clavulanic acid for three days because of cold chills and left side pain present for the last five days administered to our hospital emergency department upon the increase in his complaints. The patient was referred to the thoracic surgery clinic and hospitalized because of the effusion on the left lung detected by posteroanterior chest X-ray. The patient had no history of any chronic disease. On the physical examination, respiratory sounds were not heard on the lower left lung while the right side of the lung was clear. The body temperature, blood pressure and pulse rate were $39{ }^{\circ} \mathrm{C}, 100 / 50 \mathrm{mmHg}$ and $120 / \mathrm{min}$ respectively. In laboratory examination, hemoglobin was $11.4 \mathrm{gr} / \mathrm{dL}$ and hematocrit $34.6 \%$, white blood cell 12580 / mm3, neutrophil 11520 / $\mathrm{mm} 3$, lymphocyte $0.91 \mathrm{~mm} 3$, thrombocyte 466000 / mm3. Sedimentation rate and CRP were 89 $\mathrm{mm} / \mathrm{sec}$ and $95 \mathrm{mg} / \mathrm{dL}$, respectively. Thorax CT revealed the intensive fluid collection between visceral and parietal pleura leaves at posterior basis of the left hemithorax and parenchymal fibrotic lesions extending to pleura at the basal segments of left lobe (Figure 1.). Chest tube drainage was performed and $1500 \mathrm{cc}$ smelly and purulent fluid was evacuated from the pleural space. Imipenem therapy was started empirically.

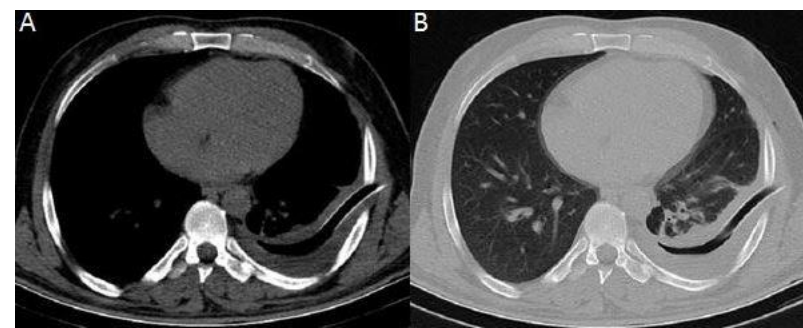

Figure 1: Axial section image of Thorax CT with $4 \mathrm{~mm}$ scan thickness without contrast material administration. (A) Mediastinum window and (B) Parenchyma window demonstrate the fluid collection with intensive contents between visceral and parietal pleura leaves and the extending drainage catheter at posterior basis of the left hemithorax

Routine biochemical, microbiological and cytological examinations were performed in the pleural fluid drained from the patient. Liquid was determined to be exudative according to Light criteria and cytological examination. PMN predominance was determined with gram staining but there were no bacteria. Sputum smear was negative for acid fast bacilli. The samples were inoculated on 5\% sheep blood agar (Merck, Darmstadt, Germany), Eosine Methylene Blue agar (EMB, Merck, Darmstadt, Germany), Sabouraud Dextrose Agar (SDA, Merck, Darmstadt, Germany) plates and brain-heart infusion broth (BHIB, Merck, Darmstadt, Germany) immediately after they arrived to the laboratory. All the agar plates and brain-heart infusion broth were incubated at $37^{\circ} \mathrm{C}$ and SDA plates were also incubated at $25^{\circ} \mathrm{C}$. The bacterium was recovered on chocolate agar, EMB agar, and 5\% blood agar as smooth colonies, with red pigment and a pungent, musty, potato like odor. The isolate was identified as $S$. rubidaea with Phoenix (Becton Dickinson Microbiology Systems, USA) fully automated identification device and the result was confirmed by conventional methods. The organism was found to be a motile gram negative cocco-bacillus. It was catalase-positive, oxidase-negative, reduced nitrates to nitrites, showed alkaline-acid with gas on triple sugar iron agar, ortho-Nitrophenyl- $\beta$ galactoside (ONPG)-positive fermented acid with adonitol, arabinose, lactose, raffinose, sucrose, and xylose. It did not ferment sorbitol and rhamnose. Indole was not produced but it utilized citrate (Procorp et al., 2016). This profile was in agreement with a good identification of $S$. rubidaea.

The strain was sent to BM Lab, Ankara, Turkey to confirm identification with sequence analysis. The 16s rDNA partial sequence of the strain sequenced with the universal primers 27F: AGAGTTTGATCMTGGCTCAG, 1492R: TACGGYTACCTTGTTACGACTT using an ABI PRISM 3130XL genetic analyzer (BM Lab, Ankara, Turkey). The CAP contig assembly software included in the BioEdit Sequence Alignment Editor 7.0.9.0 was used to modify the sequences of the BLAST (nucleotide-nucleotide) program from the National Center for Biotechnology Information (Bethesda, MD), USA). The strain 15 (3) (Gene Bank accession no: KC953862.1) was identified as S. rubidaea based on morphological characteristics and DNA sequence, which was $99 \%$ identical to the sequence of S. rubidaea NBRC 12973.

The culture antibiogram was performed according to the EUCAST (European Committee on Antimicrobial Susceptibility Testing) 2016 
(Version 6.0) Enterobacteriace tables (EUCAST, 2016). Fully automated identification device results were confirmed with Epsilometer (E-test) gradient tests (bioMerieux, France) (Table 1.).

Table 1. The antibiotics tested and the antibiogram results

\begin{tabular}{lcc}
\hline \multicolumn{1}{c}{ Antimicrobial } & $\begin{array}{c}\text { MIC } \\
\text { Concentration } \\
\text { Result (mg/L) }\end{array}$ & $\begin{array}{c}\text { Antimicrobial } \\
\text { Susceptibility } \\
\text { Test Report }\end{array}$ \\
\hline Amikacin & $\leq 8$ & $\mathrm{~S}$ \\
Ampicillin- & $\leq 4 / 2$ & $\mathrm{R}$ \\
Sulbactam & $\leq 1$ & $\mathrm{~S}$ \\
Aztreonam & $\leq 1$ & $\mathrm{~S}$ \\
Cefepime & $\leq 1$ & $\mathrm{~S}$ \\
Ceftazidime & $\leq 1$ & $\mathrm{~S}$ \\
Ceftriaxone & $\leq 0.25$ & $\mathrm{~S}$ \\
Ciprofloxacin & $\leq 0.5$ & $\mathrm{~S}$ \\
Ertapenem & $\leq 2$ & $\mathrm{~S}$ \\
Gentamicin & $\leq 2$ & $\mathrm{~S}$ \\
Imipenem & $\leq 0.5$ & $\mathrm{~S}$ \\
Levofloxacin & $\leq 2$ & $\mathrm{~S}$ \\
Meropenem & $\leq 8 / 4$ & $\mathrm{~S}$ \\
Piperacillin- & $\leq 8 / 2$ & $\mathrm{~S}$ \\
Tazobactam & & \\
Ticarcillin- & $\leq 1 / 19$ & $\mathrm{~S}$ \\
Clavulanate & & \\
Trimethoprim- & & \\
Sulfamethoxazole & &
\end{tabular}

Moxifloxacin was prescribed according to the antibiogram result. At the 48th hour of the treatment, the patient's temperature was $36^{\circ} \mathrm{C}$ and a decrease in the purulence of the drainage was observed. There was no reproduction in culture taken at the 72th hour. On the 12th day of the treatment, the chest tube was pulled out upon absence of any fluid in the drain. The treatment was completed in 21 days. The white blood cell count, the neutrophil count, CRP and sedimentation rate were $7800 / \mathrm{mm} 3,3410 / \mathrm{mm} 3,0.2 \mathrm{mg} / \mathrm{dL}$ and 19 $\mathrm{mm} / \mathrm{h}$ respectively. After normalization of the chest X-ray, the patient was discharged with full recovery.

\section{Discussion}

In this report, we discuss the clinical presentation, antimicrobial management, and outcomes of a thoracic empyema caused by Serratia rubidaea in an immunocompetent patient without underlying disease or hospitalisation history. The infection was brought under control with moxifloxacin treatment according to the antibiogram results. No treatment related adverse effects were noted. He was in a good condition and asymptomatic during follow up. We could not determine the exact mechanism of the infection.

Species in the Serratia genus which belong to the Enterobacteriacaea family are encountered rarely and are known as unusual species with the exception of Serratia marcescens and Serratia liquefaciens complex. The role of $S$. rubidaea in the pathogenesis of infectious diseases is controversial since it is rarely isolated from clinical specimens. However, although rarely, since it can be isolated from clinical specimens, this microorganism deserves further evaluation in terms of clinical significance. In the literature, there are cases such as sepsis, wound infection, urinary tract infection, endocarditis and pneumonia caused by S. rubidae (Rojo et al., 1996; Litterio et al.,2012). To our knowledge, our case is the first to present a thoracic empyema in the literature. All the cases in the mentioned reports were hospitalized and debilitated patients except for a 2-year-old child with a horse bite (Rojo et al., 1996; Litterio et al.,2012). There is no history of hospitalization, underlying disease, broad spectrum antibiotic use, or any type of invasive procedure in the history of our case, and the infection is community-based.

Carbon source utilization tests are required in the identification of 'unusual' Serratiaea spp. These tests are not a part of some common commercial identification systems and hence require special attention. Therefore, the laboratory diagnosis of these microorganisms is possibly overlooked and infections are considered as 'unusual' (Stock et al., 2003). To the best of our knowledge, S. rubidaea has not been isolated from the pleural fluid until now.

S. marcescens and many other Enterobacteriaceae express chromosomal Amp C which is reflected by resistance or decreased susceptibilities to aminopenicillins, aminopenicillin/beta-lactamase combinations, narrow-spectrum cephalosporins (e.g. cefaclor, loracarbef and cefazolin). Stock et al. determined the antibiotic susceptibilities of unusual Serratia spp. and found all species resistant to penicillin G, oxacillin, cefazolin, cefuroxime, all tested macrolides, lincosamides, streptogramins, glycopeptides, fusidic acid and rifampicin with few exceptions, and naturally sensitive to several aminoglycosides, piperacillin, piperacillin/tazobactam, carbapenems, some 
cephalosporins, fluoroquinolones and folatepathway inhibitors. They concluded that each Serratia spp. expresses its own naturally occurring Amp C $\beta$-lactamase, which might be inducible (S. ficaria, S. fonticola, S. odorifera) or non-inducible (S. rubidaea). Class A enzymes of $S$. rubidaea might be plasmid-borne, since some strains of this species were highly resistant to amoxicillin and ticarcillin. They also showed that there are differences in susceptibility to tetracyclines, the molecular basis of which is unknown. The tetracycline resistance of $S$. rubidaea is attributed to a multidrug transport system rather than a chromosomally encoded specific efflux (Tet) protein expressed at low levels (Stock et al., 2003). Our strain was susceptible to all tested antibiotics (Table 1.) The isolate was susceptible to ampicillin-sulbactam however the result was converted to resistant due to naturally occurring AmpC $\beta$-lactamase.

The natural habitat of S. rubidaea is unknown. Isolation from coconut and vegetables has been reported but it has never been associated with water, insects or other animals until now. In 2012, Litterio et al. reported that it was isolated from a mixed wound infection secondary to a horse bite and the authors concluded that the source of the infection could be grasses (Litterio et al., 2012). Our case is an agricultural worker who collects nuts. Symptoms started during hazelnut harvest and the patient applied to our hospital again in this season. The timing of hospital admission, the occupational history and susceptibility of the isolate to all tested antibiotics make us think that the source of the agent may be nature.

$S$. rubidaea is generally considered to be a rare human pathogen in immunosuppressive patients with hospitalization history. We suggest that $S$. rubidaea requires more attention when development of pleural effusion due to inadequate treatment in our immunocompetent patient and the community-based cause of the disease are considered. There is also no consensus on the natural habitat of S. rubidaea yet. Further studies are needed to identify both the clinical presentation and source of the agent.
Informed Consent: Necessary information using the patient information form and consent form was taken from the patient

Peer-review: Externally peer-reviewed.

Author Contributions: Concept- E.U., DesignE.U., Supervision E.U., Funding- E.U., A.M.S., A.Ç., C.C., A.T. Materials- E.U., Data Collection and/or Processing- E.U., A.M.S., Analysis and/or Interpretation- E.U., Literature Review- EU, Writing- E.U., Critical Review- E.U., A.M.S., A.Ç., C.C., A.T.

Conflict of Interest: No conflict of interest was declared by the authors.

Financial Disclosure: This research did not receive any specific grant from funding agencies in the public, commercial, or not for profit sectors.

\section{References}

Bonnin RA, Girlich D, Imanci D, Dortet L, Naas T. Draft Genome Sequence of the Serratia rubidaea CIP 103234T Reference Strain, a Human-Opportunistic Pathogen. Genome Announc. 2015 ;3: pii: e01340-15. doi: 10.1128/genomeA.01340-15.

Balikian JP, Herman PG, Godleski JJ. Serratia pneumonia. Radiology. 1980; 137:309-11.

Farmer III JJ, Davis BR, Hickman-Brenner FW et al. Biochemical identification of new species and biogroups of Enterobacteriaceae isolated from clinical specimens. J Clin Microbiol. 1985; 21:46-76.

Gentille D, Pérez M, Centelles MJ. Bacteremia by a Serratia rubidaea with an atypical quinolones resistance phenotype. Rev Chilena Infectol. 2014; 31:351-2.

Litterio ML, Arazi S, Hernández C, Lopardo H. Isolation of Serratia rubidaea from a mixed infection after a horse bite. Rev Argent Microbiol. 2012; 44:272-4.

Menezes EA, Cezafar FC, Andrade Mdo S, Rocha MV, Cunha FA. Frequency of Serratia sp in urine infections of intern patients in the Santa Casa de Misericórdia in Fortaleza. Rev Soc Bras Med Trop. 2004; 37:701.

Procop G.W, Church D.L, Hall G.S, Janda W.W, Koneman E.W, Schreckenberger P.C.h Koneman's Color Atlas and Textbook of Diagnostic Microbiology. Netherlands: Walters Kluwer Health, 2016. 
Rojo Ursua p, Unzaga MJ, Melero P, Iturburu I, Ezpeleta C, Cisterna R. Serratia rubidaea as an invasive pathogen. J Clin Microbiol 1996; 34: 216-7.

Stock I, Burak S, Sherwood KJ, Gruger T, Wiedemann B. Natural antimicrobial susceptibilities of strains of 'unusual' Serratia species: S. ficaria, S. fonticola, S. odorifera, S. plymuthica and $S$. rubidaea. J Antimicrob Chemother. 2003; 51:865-85.

The European Committee on Antimicrobial Susceptibility Testing. Breakpoint tables for interpretation of MICs and zone diameters. Version 7.0, 2017.Available in: http://www.eucast.org 